\title{
A Recording-Based Method for Auralization of Rotorcraft Flyover Noise
}

\author{
Nicholas M. Pera ${ }^{1}$ \\ Virginia Tech, Blacksburg, VA 24061, USA \\ Stephen A. Rizzi ${ }^{2}$ and Siddhartha Krishnamurthy ${ }^{3}$ \\ NASA Langley Research Center, Hampton, VA 23681, USA \\ Christopher R. Fuller ${ }^{4}$ \\ Virginia Tech, Blacksburg, VA 24061, USA \\ and \\ Andrew Christian ${ }^{3}$ \\ NASA Langley Research Center, Hampton, VA 23681, USA
}

Rotorcraft noise is an active field of study as the sound produced by these vehicles is often found to be annoying. A means to auralize rotorcraft flyover noise is sought to help understand the factors leading to annoyance. Previous work by the authors focused on auralization of rotorcraft fly-in noise, in which a simplification was made that enabled the source noise synthesis to be based on a single emission angle. Here, the goal is to auralize a complete flyover event, so the source noise synthesis must be capable of traversing a range of emission angles. The synthesis uses a source noise definition process that yields periodic and aperiodic (modulation) components at a set of discrete emission angles. In this work, only the periodic components are used for the source noise synthesis for the flyover; the inclusion of modulation components is the subject of ongoing research. Propagation of the synthesized source noise to a ground observer is performed using the NASA Auralization Framework. The method is demonstrated using ground recordings from a flight test of the AS350 helicopter for the source noise definition.

$\begin{array}{ll}f & =\text { frequency }(\mathrm{Hz}) \\ M & =\text { number of tail rotor harmonics } \\ N & =\text { number of main rotor harmonics } \\ p & =\text { pressure }(\mathrm{Pa}) \\ t & =\text { time }(\mathrm{s}) \\ \theta & =\text { polar (elevation) angle } \\ \psi & =\text { azimuthal (lateral) angle }\end{array}$

\section{Nomenclature}

\section{Introduction}

T $\mathrm{N}$ recent years, helicopter use in both the private and commercial sectors has seen an increase around major U.S. cities. With an increase in usage, comes an increase in noise, which can lead to increased annoyance. Annoyance has been the basis for several actions across the U.S. to limit helicopter operations ${ }^{1,2}$ and, consequently, is a focus of research by many in the rotorcraft community.

\footnotetext{
${ }^{1}$ Graduate Research Assistant, Department of Aerospace and Ocean Engineering

${ }^{2}$ Senior Researcher for Aeroacoustics, Aeroacoustics Branch, Associate Fellow AIAA

${ }^{3}$ Research Engineer, Structural Acoustics Branch, Member AIAA

${ }^{4}$ Samuel Langley Distinguished Professor of Engineering, Department of Mechanical Engineering, Associate Fellow AIAA 
Factors that lead to annoyance can fall under two main categories: acoustic and nonacoustic. An example of an acoustic factor is the impulsiveness of a sound. Age, fear and noise sensitivity are nonacoustic factors that can contribute to an individual's evaluation of a sound and its annoyance, ${ }^{3}$ e.g., the association of a helicopter as an unsafe device. The present effort is directed at the long-term goal of producing high-fidelity auralizations to help understand the acoustic factors leading to annoyance. This is motivated by the need to generate sounds of vehicles and operating conditions for which recordings do not exist. Auralization is a technique for creating audible sound files from numerical data. ${ }^{4}$ Here, it refers to the combined processes of source noise synthesis and propagation to a ground observer.

An auralization method for rotorcraft fly-in noise was previously developed for use in audibility studies. ${ }^{5}$ In the scenarios of interest, the vehicle approached from a great distance and at low altitude. The approach ended well before the overhead position. Therefore, the source emission angle was near the rotor tip path plane and did not change appreciably over the fly-in range of interest. This allowed the source noise synthesis to be based on only a single, low emission angle. Further, unsteadiness in the source and propagation path were purposefully omitted, in order that subsequent sound jury testing in the laboratory could be performed without complicating random effects. ${ }^{6}$ This was made possible by using a source noise definition that generated separate main and tail rotor pressure time histories for a single blade passage. ${ }^{7}$ For each rotor, unsteadiness of the source was removed by applying synchronous time averaging of multiple blade passages. The propagation model used was also absent of turbulence.

In a separate effort, a high-fidelity rotorcraft source noise synthesis method was developed by Hardwick, ${ }^{8}$ who adapted a method by Allen et al. ${ }^{9}$ for separating the aperiodic (modulation) components from the periodic components of turbofan tonal noise, to rotorcraft harmonic noise. Hardwick's approach successfully decomposed segments of deDopplerized noise into periodic and aperiodic amplitude and phase components, and developed a synthesis method to reconstruct the original waveform segment. Through human subject testing, he found that the inclusion of amplitude and phase modulation components increased the fidelity of the reconstructed waveform relative to signals containing only the periodic components. ${ }^{10}$ Hardwick was only able to synthesize his signals over the short time segment for which modulation data was extracted. Therefore, his approach was not amenable to auralization of a longer duration flyover.

The long-term goal of this effort is to extend these auralization capabilities to permit a full flyover and to do so in such a way that it retains unsteadiness of the source. This requires two developments: the ability to synthesize the source noise for an emission angle that changes with time and the ability to extend Hardwick's method beyond a single segment. This paper first discusses the analysis of flight test data that serve as the basis for the source noise description. It then introduces a previously developed additive synthesis method and generalizes it for emission angle dependent parameters. The resulting synthesized source noise is propagated to a ground observer using the propagation capabilities of the NASA Auralization Framework (NAF). ${ }^{11}$ The paper also discusses recent efforts to extend Hardwick's method through the development of an amplitude modulation model. ${ }^{12}$

\section{Flight Test Data Analysis}

The data used to characterize the periodic and aperiodic components of the source noise definition were derived from ground recordings of rotorcraft flyovers. This section describes the data collection and processing method, and the de-Dopplerization and segmentation process.

\section{A. Data collection and processing}

Data were obtained from a flight test ${ }^{13,14}$ of an Eurocopter AS350 BA Écureuil (more commonly known as the AStar 350 or AS350) helicopter. The AS350 has a 3-blade main rotor and a 2-blade tail rotor. It is powered by a Honeywell LTS 101-600A-3A turboshaft engine. The blade passage frequencies (BPFs) of the main and tail rotors are nominally 19 and $69 \mathrm{~Hz}$, respectively. The data were obtained from a flyover performed at Amedee Army Airfield, located at $1219 \mathrm{~m}(4000 \mathrm{ft})$ above mean sea level (MSL). The flight condition chosen is nominally a steady and level flight condition, at 80 knots indicated airspeed (KIAS) and an altitude of $91.4 \mathrm{~m}$ (300 ft) above ground level (AGL). The data are associated with flyover test run 100 and were acquired at a sampling rate of $25 \mathrm{kHz}$ with a linear microphone array positioned on the ground perpendicular to the flight path. The recorded pressure time history is shown in Figure 1 (available for download ${ }^{15}$ as audio sample S1) and its corresponding spectrogram in Figure 2. The spectrogram is shown on a magnified frequency scale in order to make the harmonics of the main and tail rotor visible. The recording was bracketed between a vehicle location approximately $610 \mathrm{~m}(2000 \mathrm{ft})$ ahead of the microphone array to a location approximately $610 \mathrm{~m}(2000 \mathrm{ft})$ aft of the array. No attempt was made to remove the effect of atmospheric absorption since the data used in this process are acquired within a short distance from the source, and because of the low frequency content of the source. 


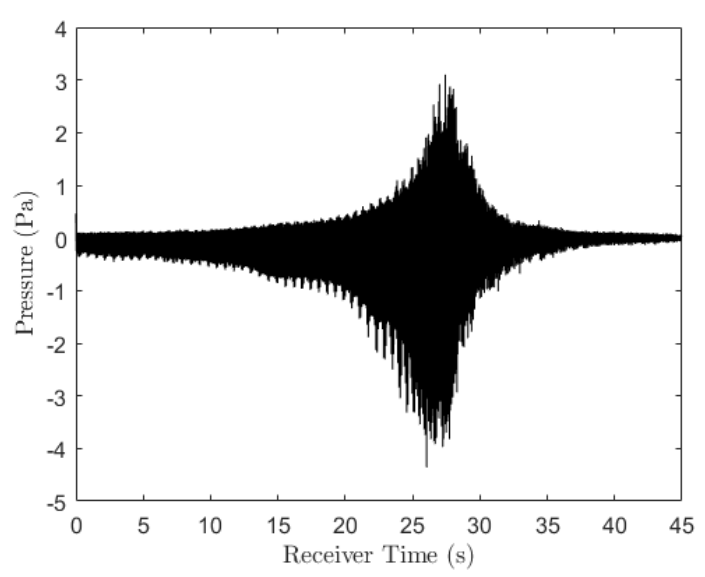

Figure 1: Measured overhead flyover made by a ground microphone.

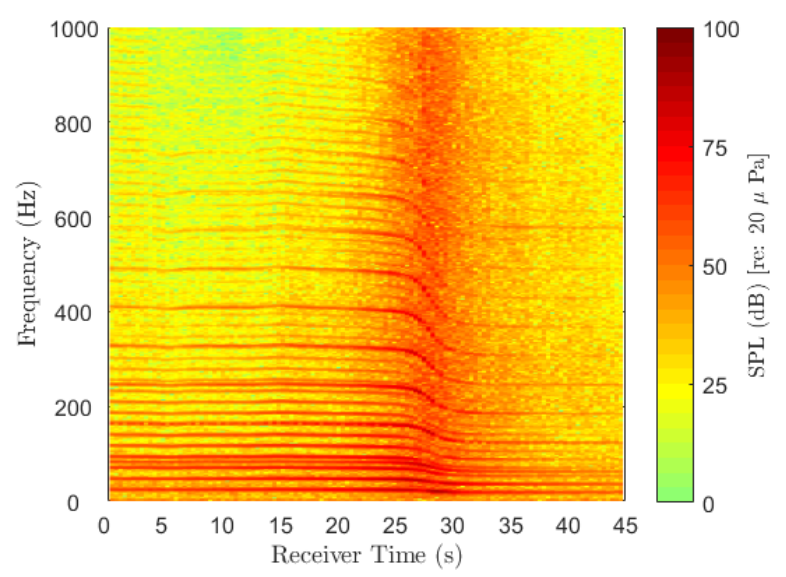

Figure 2: Spectrogram of the measured flyover shown in Figure 1.

\section{B. De-Dopplerization and segmentation process}

In order to extract the necessary periodic and aperiodic source noise components needed for synthesis, the recordings were first de-Dopplerized. The technique takes the pressure time histories recorded by the ground microphones and back propagates them to the source. The de-Dopplerization technique used was taken from Greenwood and Schmitz. ${ }^{7}$ The resulting far field signals are as if they were recorded with a virtual in-flight microphone moving with the source at a specified reference distance. In this work, a reference distance of $30.48 \mathrm{~m}$ $(100 \mathrm{ft})$, or 2.86 times the main rotor diameter, was used.

The signals are next segmented into an array of polar (or elevation), $\theta$, and azimuthal (or lateral), $\psi$, angles. The array of angles and the reference distance defines the source hemisphere. Although the method used here to divide the signals into segments also follows from Greenwood and Schmitz, this is where the similarity between that method and the present approach ends. Specifically, the Greenwood and Schmitz method goes on to separate and synchronously time-average the main and tail rotor signatures. These type of data were used in the earlier fly-in simulation. ${ }^{5}$ In the present work, each de-Dopplerized segment consists of the combined unsteady main and tail rotor noise over multiple revolutions. Following the de-Dopplerization process, the data were resampled to $10 \mathrm{kHz}$.

Because an overhead flyover was of interest, data from the microphone nearest to centerline $\left(\psi=0^{\circ}\right)$ were used. The segmented signal at a series of polar angles thus constitutes the source noise definition. Note that the convention used for emission angles was chosen to be consistent with that used by the NAF and the NASA Aircraft Noise Prediction Program (ANOPP), ${ }^{16}$ namely, $\theta=0^{\circ}$ and $180^{\circ}$ at the forward and aft polar angles, respectively, and $\psi=+90^{\circ}$ and $-90^{\circ}$ on the starboard and port sides, respectively. This convention differs from the standard used by Greenwood and Schmitz. A list of extracted polar angles and their designation by segment number is provided in Table 1. The segments are nominally contiguous (but not necessarily sample accurate), except for segment 11 . Additional details can be found in Pera. ${ }^{12}$

Table 1: Polar angles extracted from de-Dopplerization and segmentation process.

\begin{tabular}{cccc}
\hline Segment \# & Start Angle $(\mathrm{deg})$ & End Angle $(\mathrm{deg})$ & $\theta(\mathrm{deg})$ \\
\hline \hline 1 & 8.18 & 15.76 & 10.76 \\
2 & 15.76 & 25.43 & 19.50 \\
3 & 25.43 & 35.39 & 29.62 \\
4 & 35.39 & 50.10 & 41.61 \\
5 & 50.10 & 75.98 & 61.48 \\
6 & 75.98 & 109.69 & 86.11 \\
7 & 109.69 & 134.04 & 123.47 \\
8 & 134.04 & 147.75 & 141.91 \\
9 & 147.75 & 157.60 & 153.49 \\
10 & 157.60 & 166.96 & 163.44 \\
11 & 171.89 & 172.55 & 172.23 \\
\hline
\end{tabular}




\section{Source Noise Synthesis}

This section summarizes the method for synthesis of the rotor noise employing an additive synthesis technique that utilizes periodic and aperiodic data extracted from the de-Dopplerized segments. The extraction method follows that of Hardwick. ${ }^{8}$ In the following, only tonal noise from the main and tail rotor are considered.

\section{A. Rotor noise synthesis}

Synthesis of combined main and tail rotor pressure time histories is performed in the time domain using an additive synthesis approach, ${ }^{5}$ that is,

$$
p(t)=\sum_{i=1}^{N} A_{i}^{M R} \cos \left(2 \pi f_{i}^{M R} t+\phi_{o, i}^{M R}\right)+\sum_{i=1}^{M} A_{i}^{T R} \cos \left(2 \pi f_{i}^{T R} t+\phi_{o, i}^{T R}\right)
$$

in which $A$ and $\phi_{o}$ are the amplitudes and initial phases of each harmonic, $f$ are the BPFs, $N$ and $M$ are the number of main and tail rotor harmonics, respectively, and the superscripts $M R$ and $T R$ denote main and tail rotor, respectively. In this form, the amplitudes and frequencies are independent of time. The amplitudes and phases may be written in time dependent form as

and

$$
A_{i}(t)=\bar{A}_{i}+\tilde{A}_{i}(t)
$$

$$
\phi_{i}(t)=2 \pi \bar{f}_{i} t+\tilde{\phi}_{i}(t)+\phi_{o, i}
$$

in which $\bar{A}, \bar{f}$, and $\phi_{o}$ represent periodic components and the tilde quantities represent aperiodic (modulation) components. ${ }^{8}$ Note that the relationship between the phase modulation and frequency is given by

$$
\tilde{\phi}_{i}(t)=2 \pi \int_{0}^{t} \tilde{f}_{i}(\tau) d \tau .
$$

By dropping the explicit time dependence from the tilde quantities and collapsing the two summations into one with the elimination of the $M R$ and $T R$ superscripts, Eq. (1) may be rewritten as

$$
p(t)=\sum_{i=1}^{N, M}\left[\bar{A}_{i}+\tilde{A}_{i}\right] \cos \left(2 \pi \bar{f}_{i} t+\tilde{\phi}_{i}+\phi_{o, i}\right) .
$$

Reconstruction, i.e., synthesis, of the original signal thus amounts to determination of the quantities on the right hand side of Eq. (5) through a signal decomposition process.

\section{B. Signal decomposition}

The signal decomposition process was developed with the goal of preserving all of the information contained within the original signal as separate periodic and aperiodic components. This approach makes it possible to reconstruct the original signal. In contrast, Greenwood and Schmitz' method averages out the aperiodic content and, therefore, is not suitable for high fidelity signal reconstruction. The process to decompose each de-Dopplerized signal segment into its periodic and aperiodic constituents, following the method by Hardwick, ${ }^{8}$ is summarized below. It involves three steps:

1. Isolate each main and tail rotor harmonic of de-Dopplerized signal through band pass filtering.

2. For each harmonic, extract amplitude modulations, $\tilde{A}$, via Hilbert transform.

3. For each harmonic, extract phase modulations, $\tilde{\phi}$, via a simulated annealing technique.

The above processes were applied to all of the main and tail rotors harmonics (up to the Nyquist frequency of $5 \mathrm{kHz}$ ), without regard to their amplitude. Note that no attempt was made to model the aperiodic components in this process. This approach is subsequently referred to as the 'direct method.'

\section{Harmonic isolation}

Each main and tail rotor harmonic is isolated by applying a $3^{\text {rd }}$ order Butterworth band pass filter to the deDopplerized signal segment. The pass band is chosen to be equal to the main rotor BPF, and the filter is centered about the frequency of the target harmonic. When the main and tail rotor harmonics fall within the same pass band, the signal is attributed to the tail rotor. This is because the amplitudes of the lower harmonics of the tail rotor are typically higher than the amplitudes of the higher harmonics of the main rotor. In these instances, the main rotor harmonic will not contribute to the summation in Eq. (5). The filtering process is performed first in the forward direction, and then in the backward direction. This method ensures zero phase distortion and doubles the order of the 
filter to $6^{\text {th }}$ order. The result of this process is a set of fewer than $N$ pressure time histories associated with the main rotor harmonics, and $M$ for the tail rotor harmonics. These serve as input to the subsequent processing steps.

\section{Extraction of amplitude modulation}

The analytic signal is a unique complex representation of any real-valued signal and offers the benefit of direct calculation of the magnitude and phase angle at each signal sample, that is, the instantaneous values. The analytic signal of the $i^{\text {th }}$ harmonic isolated signal, $z_{i}(t)$, can be written as

$$
z_{i}(t)=x_{i}(t)+j x_{i}^{\prime}(t)=\hat{A}_{i}(t) e^{i \phi_{i}(t)}
$$

in which $x_{i}(t)$ is the harmonic isolated signal, $x_{i}^{\prime}(t)$ is the Hilbert transform of $x_{i}(t), \hat{A}_{i}(t)$ is the amplitude envelope of $x_{i}(t)$, and

$$
\hat{A}_{i}(t)=\left|z_{i}(t)\right|=\sqrt{x_{i}(t)^{2}+x_{i}^{\prime}(t)^{2}}
$$

and

$$
\phi_{i}(t)=\tan ^{-1}\left(z_{i}(t)\right)=\tan ^{-1}\left(\frac{x_{i}^{\prime}(t)}{x_{i}(t)}\right) .
$$

The amplitude modulation can be found by subtracting the mean amplitude from the amplitude envelope. Dropping the explicit time dependence, this may be written as

$$
\tilde{A}_{i}=\hat{A}_{i}-\bar{A}_{i}=\hat{A}_{i}-\frac{1}{L} \sum_{i=1}^{L} \hat{A}_{i} .
$$

in which $L$ is the number of samples in the segment. Figure 3 shows an example of an isolated main rotor tone and its associated amplitude envelope.

\section{Extraction of phase modulation}

From the phase argument in Eq. (5), the phase modulation may be written as

$$
\tilde{\phi}_{i}=\phi_{i}(t)-2 \pi \bar{f}_{i} t-\phi_{o, i} .
$$

Determination of the phase modulation thus requires the instantaneous phase $\phi(t)$ from Eq. (8) and the unknown mean frequency and initial phase. We define the line of best fit for each harmonic as $2 \pi \bar{f} t+\phi_{o}$. The goal is to find the line of best fit that minimizes the phase modulation. For any guess of the mean frequency, the initial phase estimate is given by

$$
\phi_{o, i}=\frac{1}{L} \sum_{j=1}^{L}\left(\phi_{i}\left(t_{j}\right)-2 \pi \bar{f}_{i} t_{j}\right) .
$$

This value of the initial phase assumes the phase modulation, determined from Eq. (10), has zero mean over the samples in the segment. It also reduces the problem of finding the line of best fit to be one of finding a value of the initial frequency that minimizes the phase modulation. This is done using a stochastic optimization technique called simulated annealing. ${ }^{17}$ The initial guess of the mean frequency is the nominal harmonic frequency used in the band pass filtering operation. The simulated annealing process simply searches nearby values of the mean frequency for smaller values of the phase modulation. The process will sometimes accept values of the mean frequency that increase the phase modulation. In this way, it avoids getting stuck in local minima. As the algorithm runs, the chances of it accepting such steps becomes progressively less, and it therefore converges on the desired mean frequency. Figure 4 shows the components of Eq. (10) for the case in which the line of best fit has been determined. Note that in the above, we have assumed that the difference between the mean frequency (determined through simulated annealing) and the true frequency is negligible.

\section{Signal Reconstruction}

A reconstruction of one segment of pressure time history data using Eq. (5) is next undertaken to demonstrate the effectiveness of the direct method in achieving a high-fidelity representation of the original de-Dopplerized signal. A portion of the reconstruction is shown in Figure 5, where it is seen that the reconstruction is virtually indistinguishable from the original. The root-mean-square (RMS) difference between the two signals in Figure 5 is $0.99 \mathrm{~Pa}$. In contrast, the reconstruction absent of the modulation data, that is, according to 


$$
p(t)=\sum_{i=1}^{N, M}\left[\bar{A}_{i}\right] \cos \left(2 \pi \bar{f}_{i} t+\phi_{o, i}\right)
$$

is shown in Figure 6. As expected, the average behavior is captured (RMS difference between the two signals is $1.74 \mathrm{~Pa}$ ), but the detailed behavior differs. The importance of these details can be heard be comparing the original signal (audio sample S2) with the reconstruction including modulation (audio sample S3) and the reconstruction without modulation (audio sample S4). ${ }^{15}$

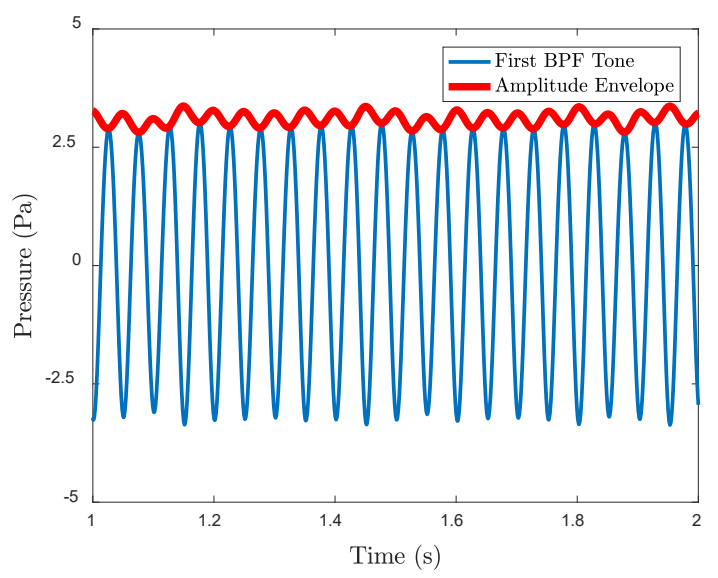

Figure 3: Isolated main rotor harmonic and its associated amplitude envelope for segment 2.

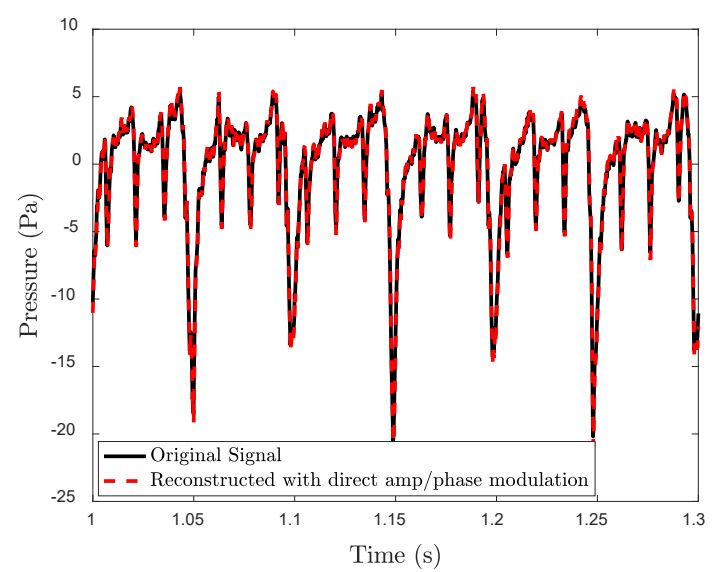

Figure 5: Original and reconstructed signals with main and tail rotor modulation for segment 2 .

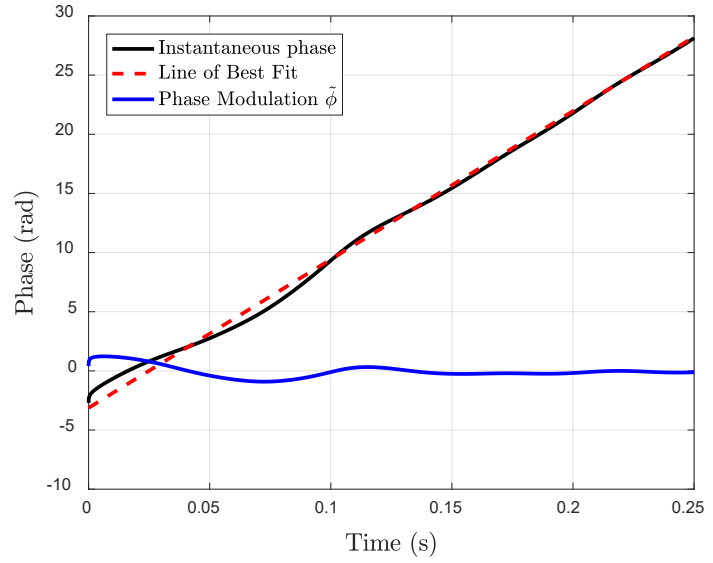

Figure 4: Result of simulated annealing process to determine phase modulation for segment 2 .

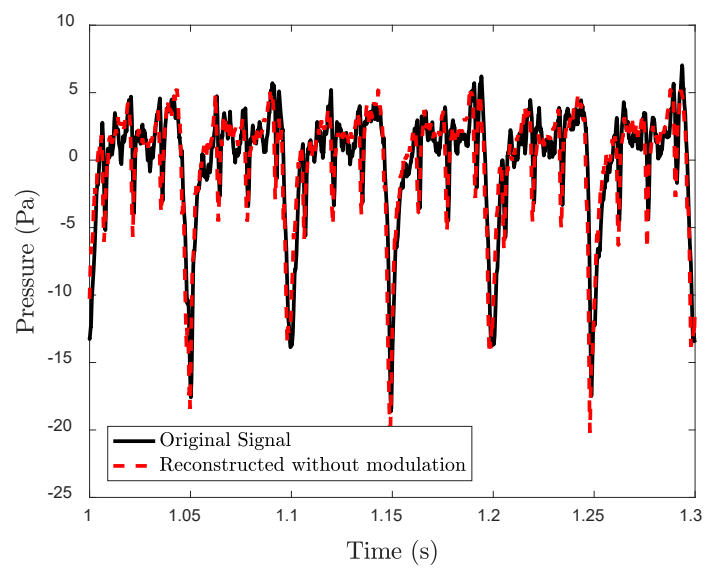

Figure 6: Original and reconstructed signals without main and tail rotor modulation for segment 2.

In order to synthesize the source noise associated with a flyover event, two additional developments are needed. The first is the ability to smoothly transition from one emission angle to the next over the course of the flyover. This will be treated in Section V of this paper. The second is the ability to extend the duration of the synthesis for as long as needed. Of course, time can be incremented indefinitely in Eq. (5), so if the amplitude and phase modulations were omitted, the second development would not be required. However, Hardwick et al. ${ }^{10}$ demonstrated through subjective testing that exclusion of the modulations detracted from the fidelity of the reconstruction. The difficulty arises because modulation data determined using the direct method is limited to the length of the segment from which it was extracted, and that length is typically small; it may be several seconds at low emission angles and less than a second at overhead angles. Hardwick attempted three different 'wrapping' techniques to extend the modulation data, but all were found to produce audible artifacts. ${ }^{8}$

We next consider a model of the amplitude modulation that allows the data to be extended indefinitely. It is expected that reconstructions using modeled amplitude modulation data will be of a lesser fidelity than using the modulation data itself (Figure 5), and of greater fidelity than using no modulation data at all (Figure 6). 


\section{Amplitude Modulation Model}

\section{A. Spectral characteristics of the amplitude modulation}

It is useful to examine the spectral characteristics of the amplitude modulations determined from the direct method to guide the development of a model. Figure 7 shows the power spectral density (PSD) of the amplitude modulation for the first few harmonics of the main rotor (recall the amplitude modulation for the fundamental is shown as the red line in Figure 3.) The roll offs of the broadband spectra steepen above roughly $10 \mathrm{~Hz}$. The presence of peaks in the spectra is attributable to leakage from neighboring tones into the pass band of the particular harmonic. The peaks occur at the difference frequencies between neighboring tones. The large peak around $12 \mathrm{~Hz}$, for example, is attributable to leakage of the first tail rotor harmonic at $69 \mathrm{~Hz}$ into the pass band of the $3^{\text {rd }}$ main rotor harmonic at $57 \mathrm{~Hz}$.

\section{B. Band-limited random model of amplitude modulation}

Based on the above observations, it was decided to model the amplitude modulation as a band-limited random process. Such an approach was successfully used by Allen et al. ${ }^{9}$ to model turbofan tone modulations. An adaptation of that approach was made here, resulting in a model with two parameters: the cutoff frequency and a scale factor. The two parameters were individually determined for each main and tail rotor harmonic, and for each section. The process for determining the model parameters is next briefly described.

Starting with the PSD of the amplitude modulation, a binary weighting vector was applied which zeroed all frequency components following the first instance of PSD dropping $30 \mathrm{~dB}$ below the maximum value. The effect of that operation is seen in Figure 8, where the weighting vector is seen to exclude the peaks due to leakage. A $3^{\text {rd }}$ order Butterworth filter magnitude response curve was chosen as the archetype shape of the model. The RMS difference between the weighted PSD and prototype filter was minimized as a function of the pass band gain and cutoff frequency. The resulting filter was applied to white noise, and a scale factor was found to match the level of the PSD of the filtered noise with that of the PSD of the amplitude modulation up to the cutoff frequency.

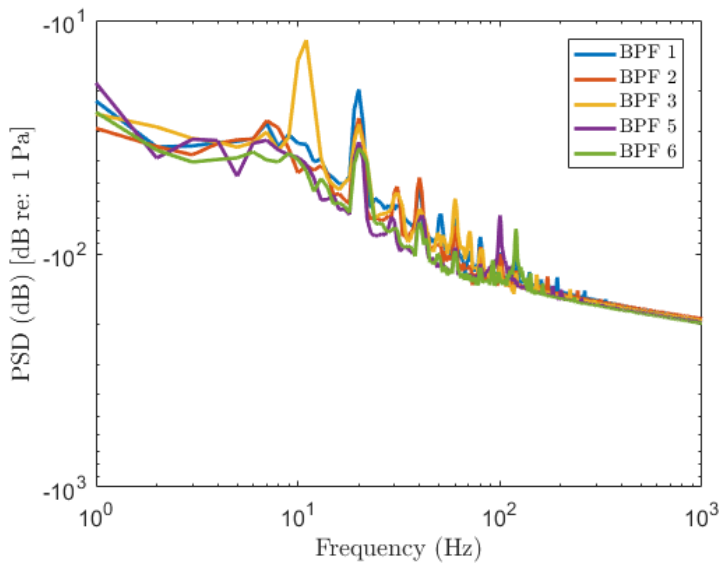

Figure 7: Power spectral density of the main rotor amplitude modulations from segment 2 .
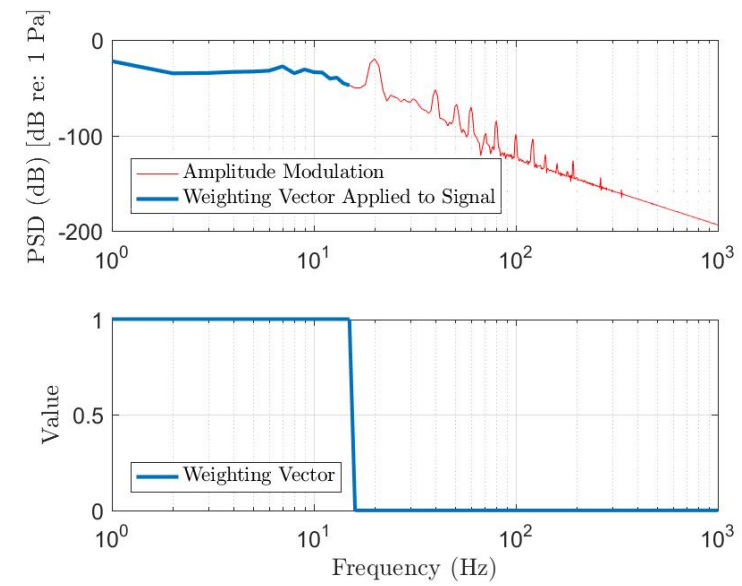

Figure 8: PSD of the main rotor amplitude modulation and its weighting vector for segment 2 .

Sample results of this model parameter estimation are shown in Figure 9 for the first harmonic of the main rotor for segment 2. Here it is seen that the PSD of the filtered signal matches the PSD of the amplitude modulation up to the cutoff frequency. The variation in cutoff frequency for most of the main and tail rotor harmonics lies in the range of $10-15 \mathrm{~Hz}$, see Figure 10. Note that there are fewer harmonics for the tail rotor up to the Nyquist frequency. The range of scale factors for main and tail rotor harmonics is shown in Figure 11. Note also that no attempt was made to predict the cutoff frequencies and scale factors from observed statistics. With these two parameters, the length of the amplitude modulation data is limited only by the length of the white noise being filtered and no longer by the length of the segment.

The effectiveness of the amplitude modulation model was assessed by reconstructing the original de-Dopplerized signal. Here the amplitude modulation data was provided by the model instead of from the direct method, that is,

$$
p(t)=\sum_{i=1}^{N, M}\left[\bar{A}_{i}+\tilde{A}_{i}^{\text {model }}\right] \cos \left(2 \pi \bar{f}_{i} t+\tilde{\phi}_{i}+\phi_{o, i}\right) .
$$


This form of the synthesis equation still used the phase modulation data obtained from the direct method. The reconstructed waveform is shown in Figure 12, and the associated sound file is provided as audio sample S5. This reconstruction is an improvement over that without any modulation (Figure 6). Although small differences are noted in the figure (RMS difference between the two signals of $1.10 \mathrm{~Pa}$ ), there is little audible difference. Reconstructions of other segments gave comparable results, indicating that this model of the amplitude modulation is also effective at other emission angles.

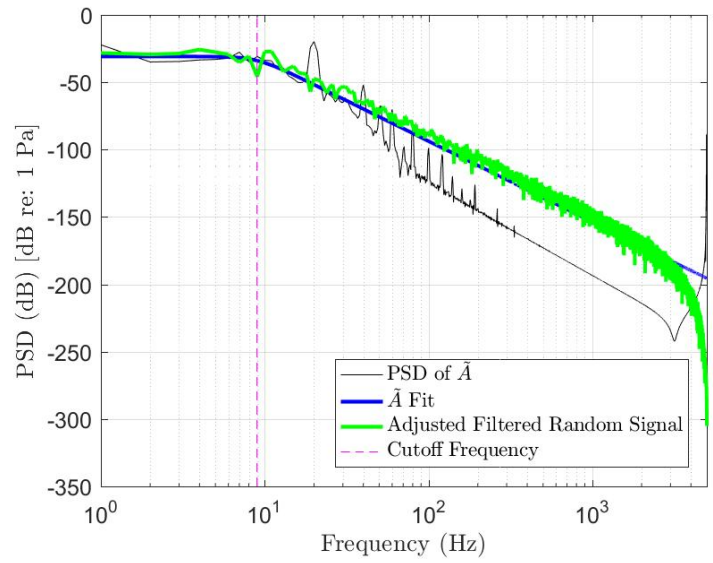

Figure 9: PSD of modeled modulation data (green) compared with original (black) and filter fit (blue).

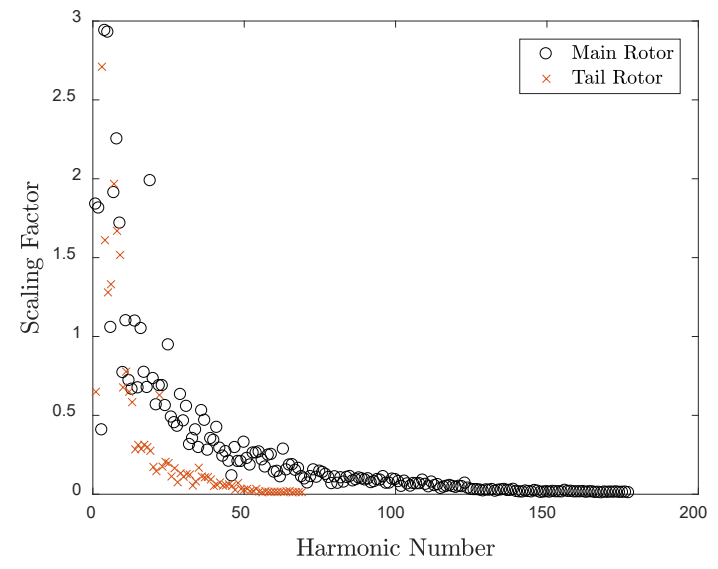

Figure 11: Main and tail rotor scale factors for harmonics of segment 2.

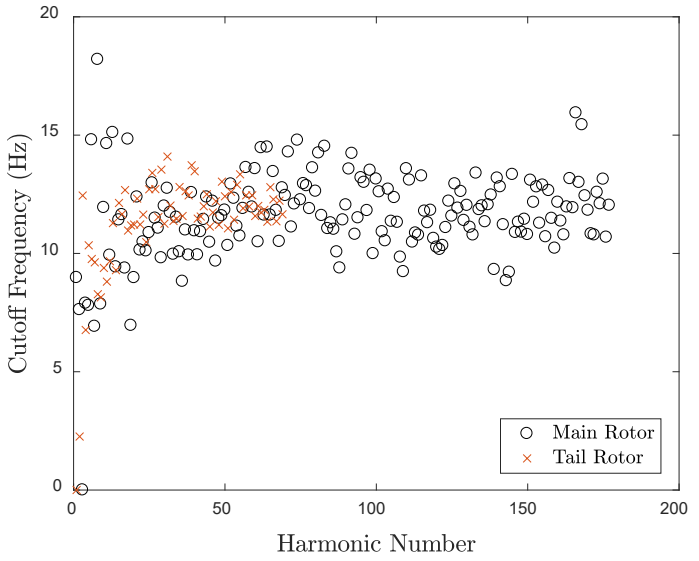

Figure 10: Main and tail rotor cutoff frequencies for harmonics of segment 2 .

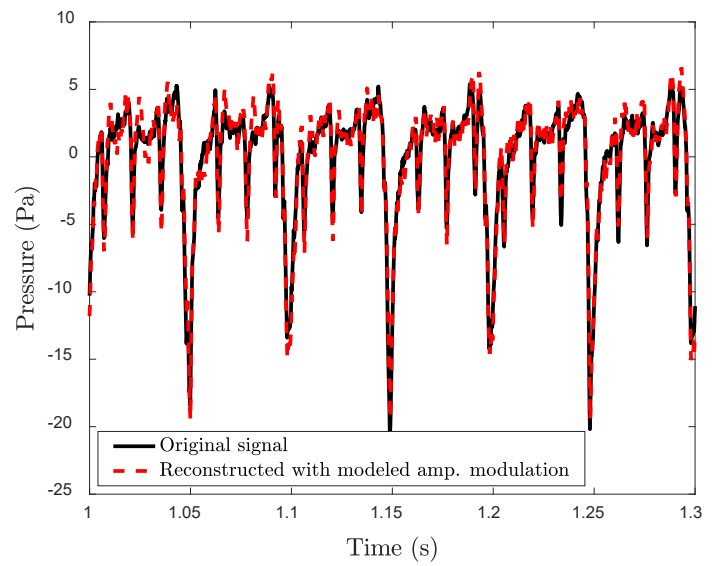

Figure 12: Original and reconstructed signals using modeled amplitude modulation for segment 2 .

Finally, note that several attempts were made to model the phase modulations. ${ }^{12}$ Unfortunately, all methods produced audible artifacts that often diminished the perceived impulsiveness. The following flyover auralizations are therefore performed without the benefit of amplitude or phase modulation in the source noise synthesis.

\section{Auralization of a Flyover}

In order to auralize a flyover event, the noise of the moving source must be synthesized, and that noise must be propagated to an observer on the ground. Each of these processes is next described.

\section{A. Source noise synthesis of a moving source}

Synthesis of the moving source noise is analogous to what a roving microphone would record while traversing the hemisphere at some fixed reference distance. This requires synthesis between segments, as the emission angles are dictated by the propagation path between the source and the observer, and these continuously change with time. For the case of an overhead flyover, the dependency on azimuthal angle vanishes. The synthesis equation of the moving source is written as 


$$
p(\theta, t)=\sum_{i=1}^{N, M}\left[\bar{A}_{i}(\theta)\right] \cos \left(2 \pi \bar{f}_{i}(\theta) t+\phi_{o, i}(\theta)\right)
$$

in which the dependency of $\phi$ on $t$ is implicit. In the above, the amplitude and phase modulation terms have been omitted. However, note that when synthesizing a moving source, the phase relationship between segments must be maintained. This is normally the case when the periodic data (amplitude, mean frequency, and initial phase) at each emission angle are the result of an acoustic calculation, for example, from Farassat's formulation $1 \mathrm{~A}^{18}$ using periodic blade loadings as input. However, no consideration of phase between segments was made in the process of segmenting the de-Dopplerized flyover data at different emission angles. Therefore, the simulated annealing method previously described must be modified to reflect the time offset of each segment $q$ within the total number of segments $Q(=11)$. To do this, we rewrite Eq. (10) for each harmonic $i$ as,

$$
\tilde{\phi}_{i, q}=\phi_{i, q}\left(t_{0, q}+t_{n, q}\right)-2 \pi \bar{f}_{i, q}\left(t_{0, q}+t_{n, q}\right)-\phi_{o, i, q}, \quad 1 \leq n \leq L
$$

in which $L$ is the number of samples in segment $q$, and $t_{0, q}$ is the time offset of the first sample of the $q^{\text {th }}$ segment, starting from recording time zero, i.e., $0 \leq t_{0,1}<t_{0,2}<\ldots<t_{0, Q}$. Taking this time offset into account changes the values of mean frequency, initial phase, and phase modulation determined from the simulated annealing process. Thus, the synthesis of the moving overhead source requires interpolation of the periodic data that properly account for the phase between segments.

\section{Interpolation of periodic data}

During the course of the flyover, the emission angle is evaluated at every sample time. Based on the emission angle, the periodic data $\left(\bar{A}, \phi_{o}\right)$ are linearly interpolated between the known segment values. In the following, the frequencies of the main and tail rotor harmonics were assigned the average value over all segments instead of being allowed to vary with emission angle, i.e., $\bar{f}_{i . q}=\bar{f}_{i}$ for all $q$. This was found to be consistent with the data and with the operation of the vehicle.

The effectiveness of the interpolation approach is dependent on how quickly the data change with emission angle (the source directivity) and the angular discretization (number of segments) of the data. Ideally, a convergence test would be performed to determine the level of discretization needed to achieve some specified accuracy for a given data set. However, this is generally not possible as the number of segments is constrained by the flight test data.

Short of a rigorous convergence test, an attempt was made to investigate the nature of interpolation errors in the periodic data. This was done by modifying the data segmentation algorithm ${ }^{7}$ to provide data in five degree increments in polar angle from $10^{\circ} \leq \theta \leq 170^{\circ}$, for a total of 33 segments. The data were interpolated between $10^{\circ}$ increments, and the interpolant was compared with the data at the intermediate angles, e.g., interpolate between the $10^{\circ}$ and $20^{\circ}$ data to an angle of $15^{\circ}$, and compare the interpolant with the data at $15^{\circ}$. The comparisons were made on the basis of the difference in the RMS pressure obtained. Pera found that differences in RMS pressure greater than $10 \%$ were limited to the shallow angles $\left(\theta \leq 20^{\circ}\right.$ and $\left.\theta \geq 160^{\circ}\right) .{ }^{12}$ Most differences were less than 5\%. Figure 13 and Figure 14 show the differences in pressure for the first 30 main rotor harmonics for $\theta=15^{\circ}$ and $\theta=40^{\circ}$, respectively. Differences in RMS pressure using interpolated (green) and direct method (black) values should be small with adequate angular discretization. The largest differences are associated with the first few harmonics of the $15^{\circ}$ data.

\section{Source noise synthesis example}

The synthesis process is demonstrated for a simulated straight and level flyover of the AS350 helicopter. In the scenario, the aircraft approaches from a distance of $1609 \mathrm{~m}(1 \mathrm{mi})$ and retreats to a distance of $1609 \mathrm{~m}(1 \mathrm{mi})$. The flight speed and altitude match the conditions under which the flight data were acquired, namely, a constant speed of 80 KIAS and altitude of $91.4 \mathrm{~m}$ ( $300 \mathrm{ft}$ ) AGL. The observer is on the ground along the centerline of the flight path. Note that the observer location is only used here to determine the emission angle, which varies from about $3^{\circ}$ to $177^{\circ}$. The source data were not extrapolated for angles less than $10.76^{\circ}$ (segment 1) and greater than 172.23 (segment 11).

In the following, Eq. (14) was used to synthesize the source pressure time history, but without taking into account the effect of the segment time offset in the simulated annealing process to determine the frequency and initial phase, as that time offset was not precisely known. Figure 15 shows the synthesized pressure time history of the source (audio sample S6). Note that in this and in subsequent figures, the source strength has been reduced by $6 \mathrm{~dB}$ to account for the pressure doubling in the ground microphone recordings. The corresponding spectrogram is provided in Figure 16. It shows the frequency of the harmonics unchanging with time. This is because the virtual microphone is traveling with the source at a fixed reference distance (hence no Doppler shift), and because the BPF was set to the average across all segments. 


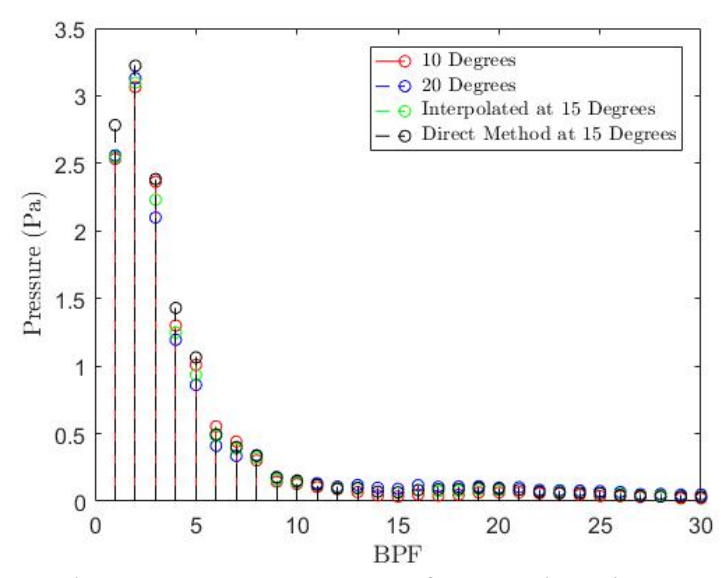

Figure 13: RMS pressure from each main rotor harmonic at $\theta=15^{\circ}$ for segment 2 .

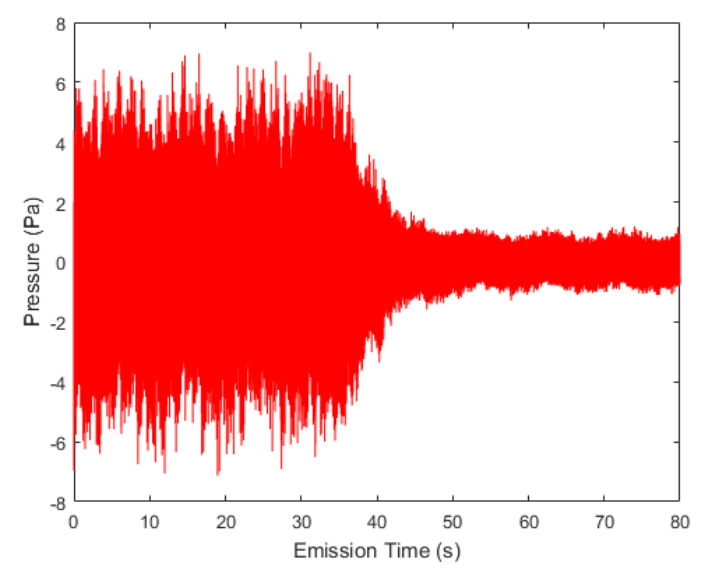

Figure 15: Source noise synthesis for segment 2.

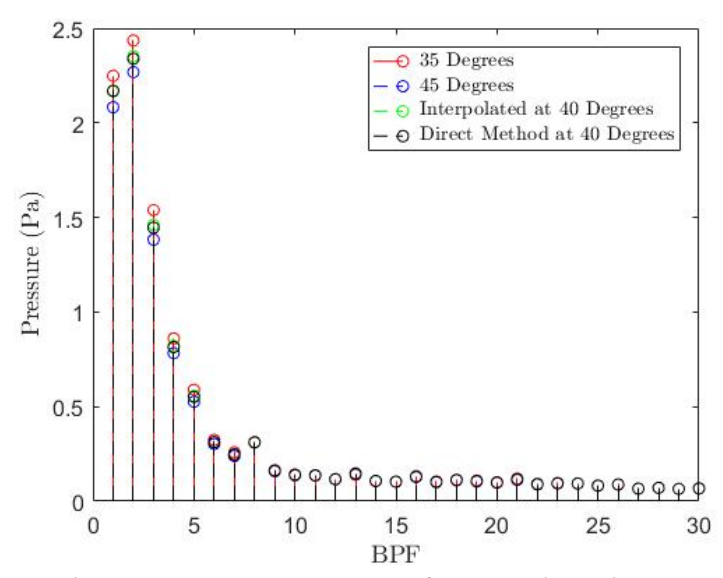

Figure 14: RMS pressure from each main rotor harmonic at $\theta=40^{\circ}$ for segment 2 .

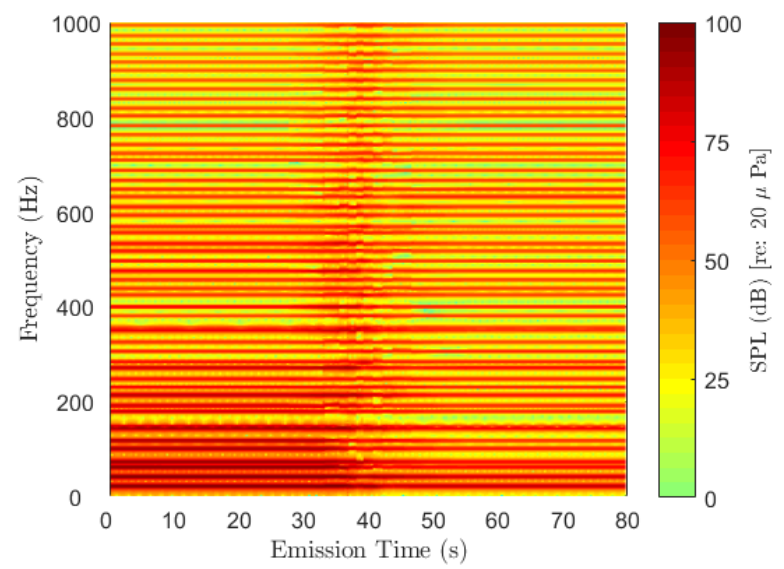

Figure 16: Spectrogram corresponding to Figure 15.

\section{Effect of phase misalignment}

Next consider the effect of phase misalignment due to neglecting the time offset in the simulated annealing process. Let $\hat{t}_{0, q}$ be an estimate of $t_{0, q}$, and let $\phi_{e}$ represent the error associated with phase estimation process. The latter may occur because the filtering method used to obtain the tone-isolated sources contains contributions from all frequencies in the pass band, and because the time history of each segment reflects multiple angles about the nominal polar angle. An estimate of the initial phase of the $q^{\text {th }}$ segment for the $i^{\text {th }}$ harmonic can then be written as

$$
\hat{\phi}_{o, i, q}=2 \pi \bar{f}_{i}\left(t_{0, q}-\hat{t}_{0, q}\right)+\phi_{o, i, q}+\phi_{e, i}
$$

The degree of misalignment in the above synthesis is unknown because the time offset of each segment is unknown. Therefore, the effect of the phase misalignment is demonstrated using a surrogate signal. The surrogate signal had no amplitude or phase modulations present. It consisted of a single rotor with a BPF of $20 \mathrm{~Hz}$. Further, it is assumed that the simulated annealing process perfectly recovered the magnitudes of all harmonics and that their frequencies were integer multiples of the BPF. A recording was simulated for an observer on the source hemisphere moving at a constant rate of $28^{\circ} \mathrm{s}$ starting from $\theta=180^{\circ}$ and ending at $0^{\circ}$. The source hemisphere was discretized into $2.5^{\circ}$ segments for a total of 73 segments. The lengths of the sections were within a sample interval of each other. The magnitude and phase of the surrogate source are shown in Figure 17 and Figure 18, respectively.

When the phase is properly aligned, $\hat{t}_{0, q}=t_{0, q}$, and the estimate of the initial phase, $\hat{\phi}_{o}$, is equal to the correct initial phase, $\phi_{o}$, plus the phase error, $\phi_{e}$. An analysis of the reconstructed source shows the phase to be close to that of the emitted signal; compare Figure 18 with Figure 19. The difference is the phase error. 
The effect of phase misalignment was simulated by setting $\hat{t}_{0, q}=0$ for all sections. The phase of the reconstructed source is dramatically different from the emitted signal; compare Figure 18 with Figure 20.

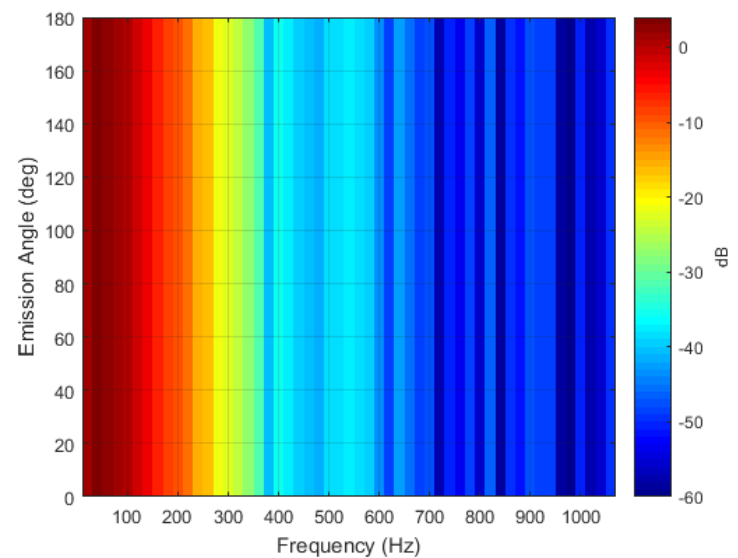

Figure 17: Magnitude of emitted signal as a function of emission angle and frequency.

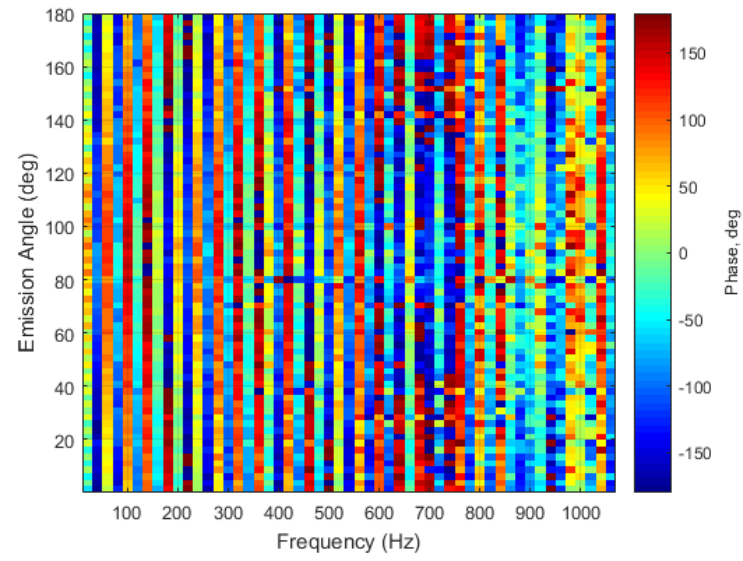

Figure 19: Phase of signal reconstructed with properly aligned phase.

Finally, the effect of phase misalignment can be seen in the reconstructed signals relative to the emitted signal. Figure 21 shows a short segment of the signal reconstructed with phase alignment to be very close to the original emitted signal, whereas the signal reconstructed with phase misalignment is markedly different. Note, however, that the reconstructed magnitudes (not shown) are the same as the original in each case. The above demonstrates the need to estimate the extraction times for each segment as accurately as possible.

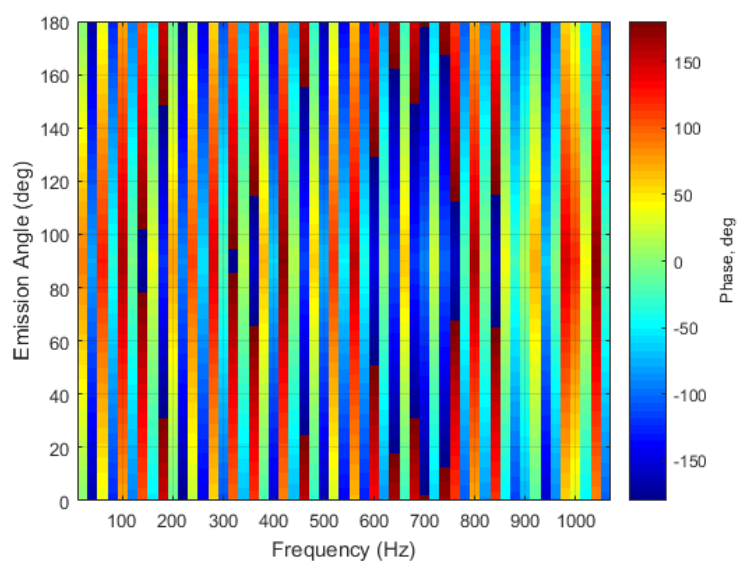

Figure 18: Phase of emitted signal as a function of emission angle and frequency.

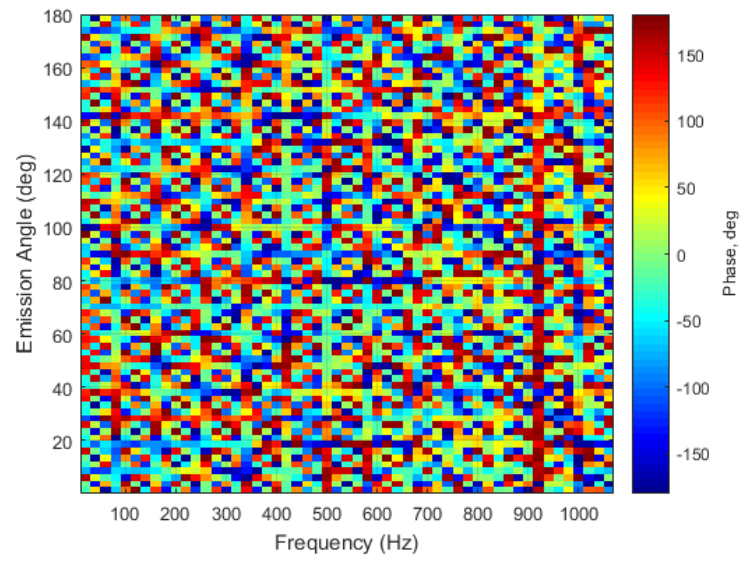

Figure 20: Phase of signal reconstructed with improperly aligned phase.

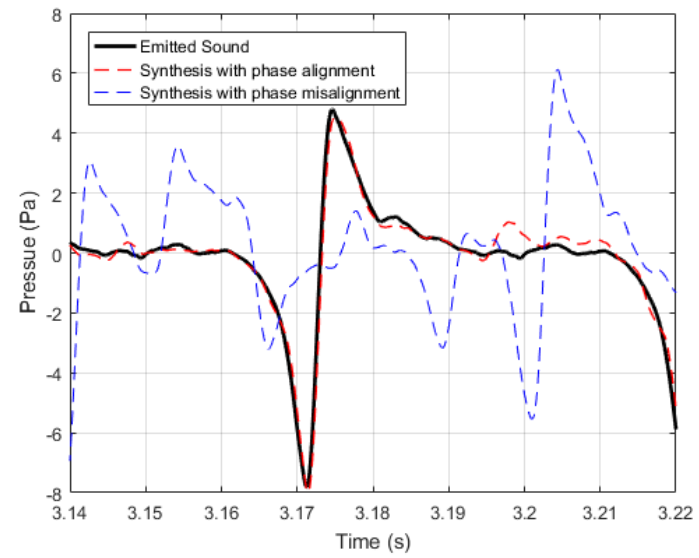

Figure 21: Comparison of signals reconstructed with and without phase misalignment relative to original. 


\section{B. Simulated Propagation}

The last stage in the auralization process entails propagation of the synthesized source noise to an observer on the ground. The process is performed in the time domain and results in a pressure time history that is referred to as a pseudo-recording. Propagation of the synthesized source noise is performed using the NAF. ${ }^{11}$ The process involves application of a gain/attenuation $(\mathrm{G})$, time delay $(\mathrm{T})$, and filter $(\mathrm{F})$, that differ between the direct path and the ground reflected path, as depicted in Figure 22. Because the path changes as a function of time (except in a hover condition), the gain, time delay and filter (GTF) change as a function of time. The GTF are specified according to physical models for spherical spreading loss $(\mathrm{G})$, absolute time delay $(\mathrm{T})$, atmospheric absorption $(\mathrm{F})$, and ground plane reflection (T, F). Each is covered in some detail in Rizzi and Sullivan, ${ }^{19}$ but are briefly summarized here. In the following, a uniform atmosphere is assumed, so the speed of sound is constant and sound propagates along a straight-line path.

Direct Path

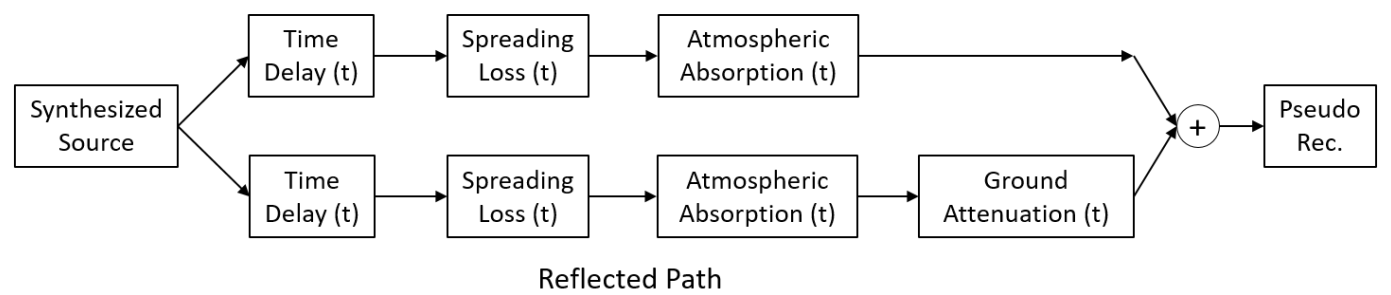

Figure 22: Block diagram showing the signal processing steps involved in generating a pseudo-recording at the observer location from the synthesized source noise.

The time-varying gain, or attenuation, is associated with spherical spreading loss. The gain is the ratio of the reference distance to the straight-line distance between the source and the observer (slant range) at the time of emission. The time delay is equal to the slant range divided by the speed of sound. Since the time delay is not usually aligned with a sample, a fractional delay line is required. Doppler shift is automatically simulated and is proportional to the time rate of change of the time delay. Atmospheric absorption is determined using a standard model and is expressed in $\mathrm{dB} /$ unit length at the 1/3-octave band center frequencies. It is accumulated along the path and converted to a minimum phase finite impulse response (FIR) filter. There are two effects related to the ground. The first relates to the difference in the time delay between the direct and ground reflected paths. When the sound is summed at the receiver, an interference pattern emerges, and this pattern changes with time as the path lengths change. The effect is referred to as a comb-filter. The second effect related to the ground has to do with its finite impedance. This frequency-dependent attenuation associated with that impedance can be expressed as an FIR filter, in which the complex reflection coefficient is determined from an impedance model.

\section{Flyover Auralization Examples}

The source noise synthesized according to the example scenario in Section V.A.2 is next propagated to the ground. Two observer locations are considered: an observer on the hard ground so that a direct comparison can be made with the recording, and an observer at a $1.2 \mathrm{~m}(4 \mathrm{ft})$ height above a grass surface. The atmosphere was taken as uniform at a temperature of $2.8^{\circ} \mathrm{C}$. Since atmospheric absorption was not taken out of the original signal during the deDopplerization process, its effect is already reflected in the source noise synthesis. Therefore, an atmospheric absorption filter was not applied. For the grass surface, a 4096 tap ground impedance filter was obtained using the Delany-Bazley model ${ }^{20}$ with a flow resistivity of $200 \mathrm{kPa}-\mathrm{s} / \mathrm{m}^{2}$ (see Embleton and Daigle ${ }^{21}$ ). Plane wave propagation was assumed in the ground impedance calculation.

Figure 23 shows a comparison of the ground recording from Figure 1 with the auralized flyover (audio sample S7) at the ground observer location. The roughly $5 \mathrm{~s}$ delay in the beginning of the auralized signal reflects the propagation time from the initial position $1609 \mathrm{~m}$ ( $1 \mathrm{mi}$.) away. The difference between the synthesized source noise in Figure 15 and the propagated sound reflects the range dependent spreading loss and time delay. The comparison in Figure 23 is reasonable, given that no attempt was made to correct for the actual path over the microphone, and given the coarseness of the data in the overhead region where the differences are the greatest. The contribution of phase misalignment to this disparity is not known. The differences are greatest on the negative pressure side. The spectrogram of the auralized signal, Figure 24, clearly shows the Doppler shift resulting from the change in the time delay. As well, the reduction in amplitude as a function of range is apparent through comparison with Figure 16.

The greatest utility of performing an auralization is for generating flyover noise for conditions where recordings do not exist. This is demonstrated for the case of the $1.2 \mathrm{~m}$ observer in which the ground impedance has been changed from the original recording. It is seen in Figure 25 that the amplitude of auralized signal for the $1.2 \mathrm{~m}$ observer is 
reduced relative to that of the ground observer. The spectrogram shown in Figure 26 reveals the interference between the direct and ground reflected paths, and this effect is clearly heard in audio sample S8.

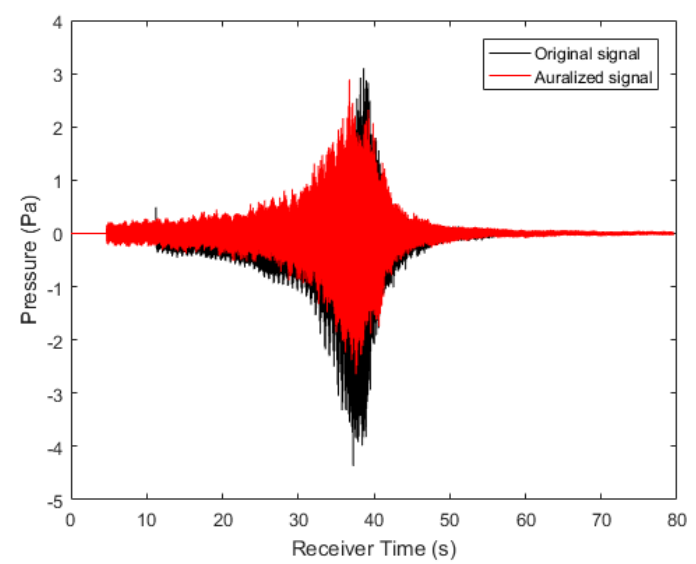

Figure 23: Comparison of ground recording with auralized signal.

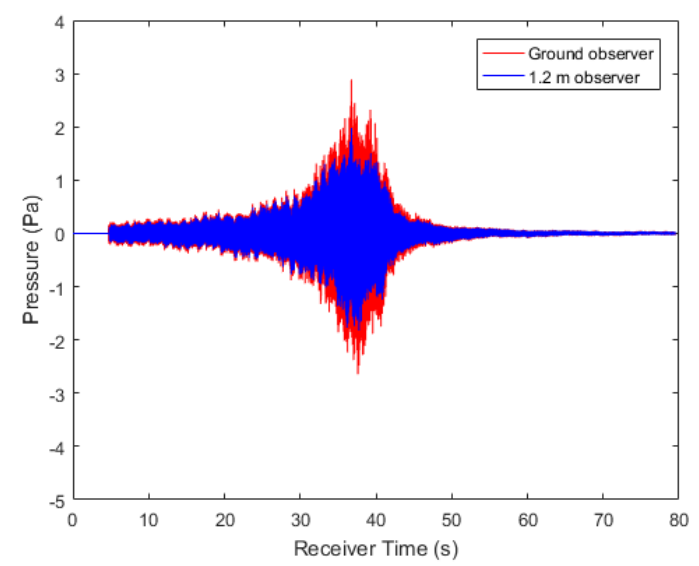

Figure 25: Comparison of auralized signals at ground and $1.2 \mathrm{~m}$ observers.

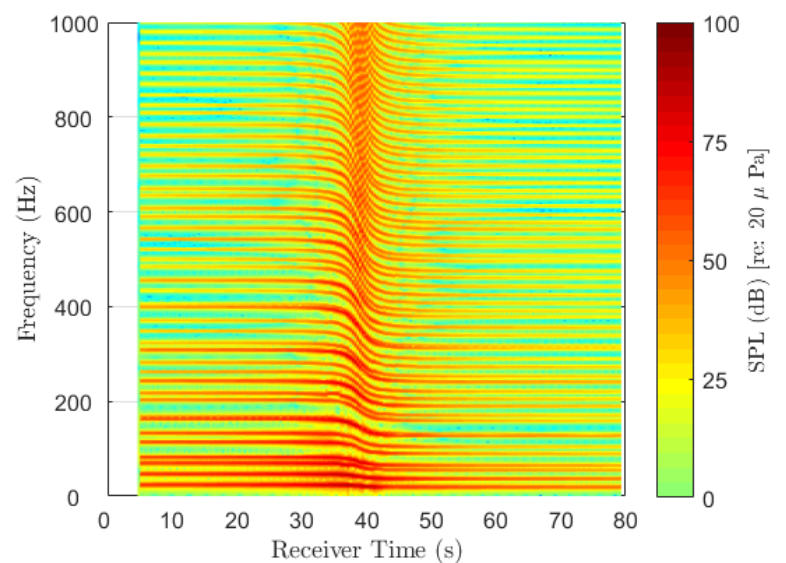

Figure 24: Spectrogram of auralized signal.

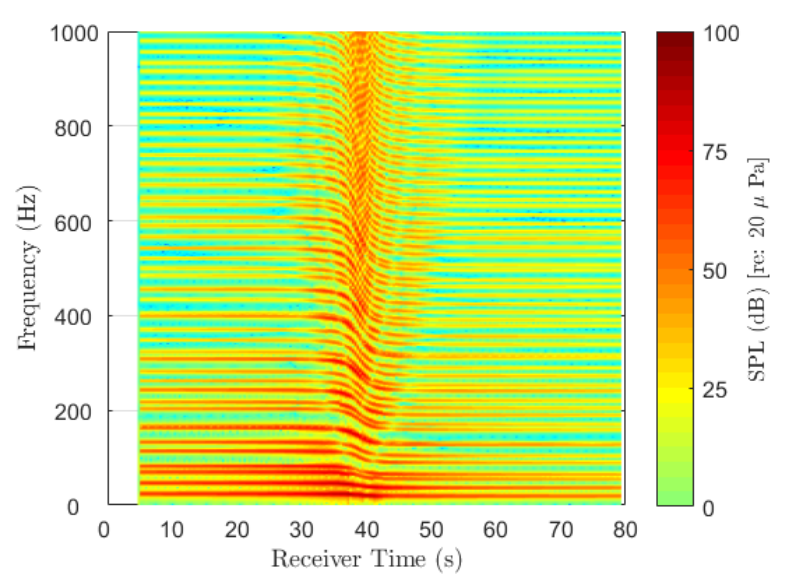

Figure 26: Spectrogram of auralized signal at $1.2 \mathrm{~m}$ observer.

\section{Conclusions}

A method to auralize rotorcraft flyover noise has been developed. The method uses a source noise description based on ground recordings of flyover data. This allows the source description to be decomposed into periodic and aperiodic (modulating) amplitude and phase components. A model of the amplitude modulation was developed and shown to be effective in reconstructing de-Dopplerized source noise segments. The inclusion of that modulation component into the auralization of a full flyover is on hold pending the development of a model for the phase modulation. Auralizations were made of an AS350 flyover for observers on a hard ground and above a grass surface. Differences were noted between the recorded flyover data and the auralization for the observer on the ground.

There are areas for improvement in the flyover auralization beyond the inclusion of amplitude and phase modulation. An estimation of the sensitivity of the generated pseudo-recordings to variances in the flight path is needed to determine the importance of including that effect. The auralization process can likely benefit from greater resolution data on the source hemisphere. One means of doing so might be to analyze the de-Dopplerized signal for periodic amplitude and phase at a higher resolution, and the amplitude and phase modulation (which require more data) at a coarser resolution. The effect of this on the auralized signal remains to be determined. Additionally, changes to the segment extraction algorithm need to be made to ensure that the extraction times are accurately captured for phase alignment. Finally, beyond quantitative measures, an evaluation of the fidelity of the auralization vis-à-vis the original signal through human subject testing would be worthwhile. 


\section{Acknowledgments}

This work was performed with support from the Revolutionary Vertical Lift Technology Project of the NASA Advanced Air Vehicles Program.

\section{References}

1"Report on the Los Angeles Helicopter Noise Initiative," Federal Aviation Administration 2013.

2"The New York North Shore Helicopter Route," Federal Aviation Administration, Department of Transportation, 14 CFR Part 93, Docket No. FAA-2010-0302, 2010.

${ }^{3}$ Basner, M., Clark, C., Hansell, A., Hileman, J., Janssen, S., Shepherd, K., and Sparrow, V., "Aviation Noise Impacts: State of the Science," Noise and Health, Vol. 19, No. 87, 2017, pp. 41-50.

${ }^{4}$ Vorländer, M., Auralization - Fundamentals of acoustics, modelling, simulation, algorithms and acoustic virtual reality. SpringerVerlag, Berlin, 2008.

${ }^{5}$ Rizzi, S.A. and Christian, A., "A method for simulation of rotorcraft fly-in noise for human response studies," InterNoise 2015, San Francisco, CA, 2015.

${ }^{6}$ Rizzi, S.A., Christian, A.W., and Rafaelof, M., "A laboratory method for assessing audibility and localization of rotorcraft fly-in noise," AHS Forum 73, Paper 101, Fort Worth, TX, 2017.

${ }^{7}$ Greenwood, E. and Schmitz, F.H., "Separation of main and tail rotor noise from ground-based acoustic measurements," AIAA Journal of Aircraft, Vol. 51, No. 2, 2014, pp. 464-472.

${ }^{8}$ Hardwick, J.R., "Synthesis of rotorcraft noise from flyover data," M.S. Thesis, Department of Mechanical Engineering, Virginia Tech, Blacksburg, VA, 2014.

${ }^{9}$ Allen, M.P., Rizzi, S.A., Burdisso, R., and Okcu, S., "Analysis and synthesis of tonal aircraft noise sources," 18th AIAA/CEAS Aeroacoustics Conference, AIAA-2012-2078, Colorado Springs, CO, 2012.

${ }^{10}$ Hardwick, J.R., Christian, A., and Rizzi, S.A., "Evaluation of the perceptual fidelity of a novel rotorcraft noise synthesis technique," The Journal of the Acoustical Society of America, Vol. 136, No. 4, 2014, pp. 2287-2287.

${ }^{11}$ Aumann, A.R., Tuttle, B.C., Chapin, W.L., and Rizzi, S.A., "The NASA Auralization Framework and plugin architecture," InterNoise 2015, San Francisco, CA, 2015.

${ }^{12}$ Pera, N.M., "Development of a method for analysis and incorporation of rotorcraft fluctuation in synthesized flyover noise," M.S. Thesis, Department of Aerospace and Ocean Engineering, Virginia Tech, Blacksburg, VA, 2017.

${ }^{13}$ Stephenson, J.H. and Greenwood, E., "Effects of vehicle weight and true versus indicated airspeed on BVI noise during steady descending flight," AHS Forum 71, Virginia Beach, VA, 2015.

${ }^{14}$ Watts, M.E., Greenwood, E., Sim, B., Stephenson, J.H., and Smith, C.D., "Helicopter acoustic flight test with altitude variation and maneuvers," NASA TM-2016-219354, 2016.

15"Aircraft flyover simulation," https://stabserv.larc.nasa.gov/flyover/, NASA, 2018.

${ }^{16}$ Zorumski, W.E., "Aircraft noise prediction program theoretical manual," NASA TM-83199, 1982.

${ }^{17}$ Kirkpatrick, S., Gelatt, C.D., and Vecchi, M.P., "Optimization by simulated annealing," Science, Vol. 220, No. 4598, 1983, pp. 671-680.

${ }^{18}$ Farassat, F., "Derivation of formulations 1 and 1A of Farassat," NASA TM-2007-214853, 2007.

${ }^{19}$ Rizzi, S.A. and Sullivan, B.M., "Synthesis of virtual environments for aircraft community noise impact studies," 11th AIAA/CEAS Aeroacoustics Conference, AIAA-2005-2983, Monterey, CA, 2005.

${ }^{20}$ Delany, M.E. and Bazley, E.N., "Acoustical properties of fibrous absorbent materials," Applied Acoustics, Vol. 3, No. 2, 1970, pp. 105-116.

${ }^{21}$ Embleton, T.F.W. and Daigle, G.A., Aeroacoustics of Flight Vehicles: Theory and Practice. Volume 2: Noise Control, NASA RP 1258, Atmospheric Propagation, H.H. Hubbard, Editor. 1991. 